\title{
Complete anatomic reduction and monosegmental fusion for lumbar spondylolisthesis of Grade II and higher: use of the minimally invasive "rocking" technique
}

\author{
Deshpande V. Rajakumar, MCh, Akshay Hari, DNB, Murali Krishna, MCh, Ankit Sharma, DNB, and \\ Manjunatha Reddy, MBBS
}

Department of Neurosurgery, Fortis Hospitals, Bangalore, India

OBJECTIVE Different surgical approaches have been described for treatment of spondylolisthesis, including in situ fusions, reductions of various degrees, and inclusion of healthy adjacent segments into the fusion construct. To the authors' knowledge, there are only sparse reports describing consistent complete reduction and monosegmental transforaminal lumbar interbody fusion for spondylolisthesis using a minimally invasive technique. The authors assess the efficacy of this technique in the reduction of local deformity and correction of overall sagittal profile in single-level spondylolisthesis.

METHODS This cohort study consists of a total of 36 consecutive patients treated over a period of 6 years. Patients with varying grades of lumbar spondylolisthesis (29 Meyerding Grade II and 7 Meyerding Grade III) were treated with operative reduction via minimally invasive transforaminal lumbar interbody fusion (MIS-TLIF) in which the "rocking" technique was used. The clinical outcomes were measured using the visual analog scale (VAS) for pain and the Revised Oswestry Disability Index (ODI) for low-back pain/dysfunction. Meyerding grade, pelvic incidence (PI), lumbar lordosis (LL), disc space angle (DSA), pelvic tilt (PT), and sacral slope (SS) were assessed to measure the radiological outcomes. These were reviewed for each patient for a minimum of 2 years.

RESULTS At most recent follow-up, $94 \%$ of patients were pain free. There were 2 patients (6\%) who had moderate pain (which corresponded to higher-grade of listhesis), but all showed an improvement in pain scores $(p<0.05)$. The mean VAS score improved from 6.5 (SD 1.5) preoperatively to 1.6 (SD 1.3) and the mean ODI score improved from 53.7 (SD 13.1) preoperatively to 22.5 (SD 15.5) at 2-year follow-up.

All radiological parameters improved following surgery. Most significant improvement was noted for LL, DSA, and SS. Both LL and SS were found to decrease, while DSA increased postoperatively. PI remained relatively unchanged, while PT showed a mild increase, which was not significant. Good fusion was achieved with implants in situ at 2-year followup. A $100 \%$ complete reduction of all grades of spondylolisthesis was achieved. The overall sagittal profile improved dramatically. No major perioperative complications were encountered.

CONCLUSIONS Minimally invasive monosegmental TLIF for spondylolisthesis reduction using this rocking technique is effective in the treatment of various grades of spondylolisthesis. Consistent complete reduction of the slippage as well as excellent correction of overall sagittal profile can be achieved, and the need for including healthy adjacent segments in the fusion construct can be avoided.

https://thejns.org/doi/abs/10.3171/2017.5.FOCUS17199

KEY WORDS minimally invasive; transforaminal lumbar interbody fusion; spondylolisthesis; high grade; monosegmental fusion; complete reduction; rocking technique

$\mathrm{M}$ ANAGEMENT of patients with spondylolisthesis, in terms of indications and technique, can be difficult, especially in those with higher grades of spondylolisthesis. Associated with this, the degree of surgical reduction of the slippage is difficult to achieve consistently.
The prevalence of disc degeneration in patients with spondylolisthesis and symptoms of low-back pain are related to the instability of the involved segment., ${ }^{67,32}$ The instability may cause transfer of mechanical forces to the surrounding vertebral endplates and associated discs. ${ }^{28}$ Some authors have even suggested that the degree of disc

ABBREVIATIONS BOP = break-off plug; DSA = disc space angle; LL = lumbar lordosis; MIS = minimally invasive surgery; ODI = Oswestry Disability Index; PEEK = polyetheretherketone; $\mathrm{PI}$ = pelvic incidence; $\mathrm{PT}$ = pelvic tilt; $\mathrm{SS}$ = sacral slope; TLIF = transforaminal lumbar interbody fusion; VAS = visual analog scale.

SUBMITTED April 2, 2017. ACCEPTED May 19, 2017.

INCLUDE WHEN CITING DOI: 10.3171/2017.5.FOCUS17199. 
degeneration at the spondylolisthesis-affected level is a more important factor in the choice of fusion procedure than the age of the patient or the grade of vertebral slip. ${ }^{6}$ However, there are other causes of the pain, including associated facet joint arthritis and stresses on the ligaments, which contain sensory nerve fibers.

Investigations into the sagittal spinal alignment have increased since the importance of pelvic incidence (PI) was realized. ${ }^{19,30}$ Sagittal alignment plays an important role in the development of many spinal disorders. ${ }^{1,9}$ Furthermore, there is mounting evidence in the literature showing the close relationship between elevated PI and the progression and severity of spondylolisthesis. ${ }^{10,13,16,20,25}$

Therefore, the most important indications for surgery in spondylolisthesis become relief of pain and correction of the segmental deformity. ${ }^{3}$ Various surgical approaches have been described for treating spondylolisthesis, including in situ fusions, partial reduction, and inclusion of healthy adjacent segments in the fusion construct. ${ }^{29,31}$ However, the best treatment option for spondylolisthesis (Grade II and higher) remains uncertain. To our knowledge, there have been sparse reports describing complete reduction and monosegmental transforaminal lumbar interbody fusion (TLIF) for higher grades of spondylolisthesis using minimally invasive approaches.

We describe a minimally invasive approach for complete anatomic reduction of spondylolisthesis, Grade II and higher, in which we have used a novel "rocking" technique to achieve consistent results.

\section{Methods}

\section{Patient Selection and Demographic Information}

This is a prospective cohort study of 36 consecutive patients with varying grades of single-level spondylolisthesis (Meyerding II and III) ${ }^{21}$ who underwent minimally invasive monosegmental TLIF] All surgeries were performed by the senior corresponding author (D.V.R.) during a 6-year period from 2009 to 2015, at Fortis Hospital, Bangalore, and all patients were followed up for a minimum of 2 years. Patients chosen were those with a diagnosis of low-back pain with or without sciatica, with symptoms correlating to imaging findings (preoperative MRI and dynamic lumbar radiography confirming instability with loss of sagittal balance), and with symptoms refractory to conservative management. Exclusion criteria were previous back surgery, multilevel disease, and pathological conditions such as spinal malignancy, infection, or trauma.

Quantitative clinical data were collected with the use of two preoperative back pain-scoring instruments - the visual analog scale (VAS) for pain and the revised Oswestry Disability Index (ODI). ${ }^{8}$

Radiological data included measurement of the preoperative sagittal balance parameters on radiographs-i.e., Meyerding grade, lumbar lordosis (LL), disc space angle (DSA), sacral slope (SS), pelvic tilt (PT), and PI (which was defined by the following equation: $\mathrm{PI}=\mathrm{SS}+\mathrm{PT}$ ). Accordingly, the pelvis was termed as "balanced" if there was a high SS and low PT and "unbalanced" if there was high PT and low SS, as described by Hresko et al. ${ }^{13}$ DSA was considered as the angle subtended by a line along the lower border of the superior vertebra and the upper border of the inferior vertebra. Lordotic angles were recorded as positive and kyphotic angles were noted as negative.

For the evaluation of interbody fusion, at the final follow-up observation, the fusion rate was analyzed by applying the anterior fusion grade described by Bridwell et al. (Grade I: fusion with remodeling and trabeculae; Grade II: graft intact, not fully remodeled, no radiolucency; Grade III: graft intact, but a definite lucency; and Grade IV: definitely not fused, collapse). ${ }^{5}$ We considered anterior fusions of Grades I and II to represent "good" fusions.

The clinical and radiological outcomes were analyzed preoperatively and reviewed for each patient postoperatively (immediate after surgery, at 6 months, 1 year, and 2 years). Statistical analysis was performed using Wilcoxon's test for clinical assessment and paired t-tests for radiological assessment. A probability value $<0.05$ was regarded as significant. Association between the change in clinical and radiological parameters was performed by using Fisher's exact test.

\section{Operative Procedure}

The procedure was performed after induction of general anesthesia, and each patient received a single dose of prophylactic antibiotics at the time of skin incision. The patient was then placed prone in a neutral position on the radiolucent operating table to maintain the natural lumbar lordosis. In many cases, such a position under anesthesia was itself enough to bring about some degree of reduction in the slippage. Proper positioning also ensured that the spine was parallel to the floor. On true lateral fluoroscopy, the pedicles would overlie one another with the endplates appearing linear and not elliptical.

Under fluoroscopic guidance, the appropriate level was confirmed and a 2- to $2.5-\mathrm{cm}$ incision was made approximately 3.5 to $4 \mathrm{~cm}$ lateral to the midline, on the side of pain, and deepened up to the fascia (Fig. 1).

Using sequential tubular dilators, a transmuscular approach was used to "dock" onto the facet joint through the "Wiltse plane" over which the Quadrant retractor system (Medtronic), of required length, was introduced (Fig. 2). The retractor was then attached to a flexible arm fixed to the side of the operating table. The superoinferior and mediolateral portions of the retractor blades were adequately expanded and the muscles dissected, allowing access to the affected area of the lumbar spine, including the facet joint, pars interarticularis, and surrounding area (Fig. 3).

The anatomical localization was reconfirmed using Carm fluoroscopy. The subsequent steps were performed under microscopic visualization. The ipsilateral facet joint, pars interarticularis, and lamina were identified after removing soft tissue. Using a bone chisel, cuts were made at the mid-pars level superiorly and medially over the lamina, just medial to the facet joint (Fig. 4). The inferior articular process would then be freely floating and easily removed. Part of the superior articular process of the inferior vertebra was amputated to make it flush with the superior border of the pedicle. All bone fragments were retained for use as an autograft during further interbody fusion. Once the ligamentum flavum was identified and 


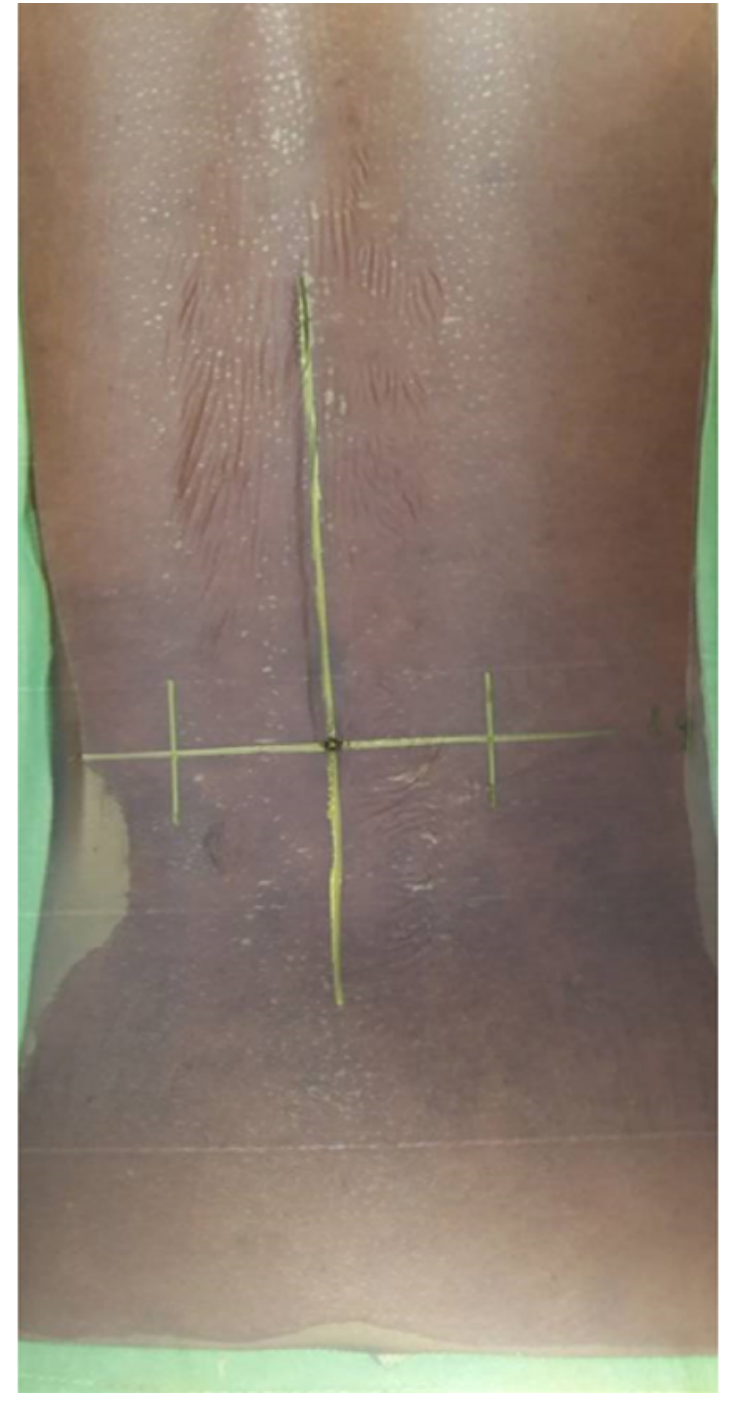

FIG. 1. Patient positioning and marking of the incision.

removed, the dura and nerve roots would become decompressed. Access to the disc space was achieved through Kambin's triangle (bordered medially by the traversing nerve root, superolaterally by the exiting nerve root, inferiorly by the pedicle), and complete discectomy was performed using specialized disc-removal instruments. In all cases, the endplate was sufficiently curetted, and the anterior annulus was identified. It was felt that it would be easier to reduce the slipped vertebral body once adequate decompression and distraction were achieved. After this, the correct spacer size was confirmed using interbody trial spacers, which were then retained in place. This was done to keep the disc space distracted and assist in the rocking technique used for reduction.

\section{Reduction-the Rocking Technique}

For this technique, initially, percutaneous screws were inserted on the contralateral side, which was done using the CD Horizon Sextant system (Medtronic) (Fig. 5).

The Sextant towers were then mated. Occasionally, be- cause of the degree of spondylolisthesis, the mating of the towers would occur at an acute angle related to the skin, requiring some force. Once the two towers were aligned with each other, the entire system was gently rocked back and forth a few times, in the craniocaudal direction, to release the involved segment from any remaining soft-tissue attachments (Fig. 6, Video 1).

VIDEO 1. Operative video showing the rocking technique. Copyright Deshpande V. Rajakumar. Published with permission. Click here to view.

This movement would then gradually reduce the listhesis, which was confirmed under fluoroscopy (Fig. 7, Video 2).

VIDEO 2. Fluoroscopic video of the rocking technique. Copyright

Deshpande V. Rajakumar. Published with permission. Click here to view.

The towers were held together firmly such that the vertebrae were in the reduced position. An appropriately sized pre-curved lordotic rod was then introduced, through a separate incision using the Sextant arc, into the pedicle screw heads that were held by the towers (Fig. 8).

The screw cap/break-off plug (BOP) was tightened first over the screw of the lower nondisplaced vertebra such that it would behave like a monoaxial screw. By pivoting the rod against the screw placed into nondisplaced lower vertebra, the screw attached to the slipped vertebra gets lifted up, thus reducing the spondylolisthesis. Once adequate reduction was confirmed fluoroscopically, the BOP was placed over the reduced screw and complete tightening was achieved. The rocking movement thus helped the slipped vertebra to gradually come into position.

In rare cases, if complete reduction was still not achieved, bilateral facetectomy was performed using Quadrant retractors bilaterally, after which the rocking technique invariably brought about satisfactory reduction.

\section{Interbody Fusion}

Once the listhesis was corrected, attention was brought back to the Quadrant side. The trial spacer was removed and the prepared disc space was packed with bone graft adequately along the anterior portion. A polyetheretherketone (PEEK) cage filled with bone graft was then inserted for anterior intervertebral structural support (Fig. 9).

Through the same exposure, ipsilateral pedicle screw/ rod fixation was performed under direct visualization. Once again, the BOP was first placed in the lower screw in extension so that the rod was lifted (Fig. 9). Then, using specialized rod reducers, the superior screw was held and gently pulled, thereby correcting any further slippage that remained, and the second BOP was subsequently placed.

The placement and positioning of implants were confirmed intraoperative using C-arm fluoroscopy. The surgical wound was closed using subcutaneous absorbable sutures.

\section{Postoperative Protocol}

Patients were ambulatory and dressings were changed on the 1st postoperative day. Discharge was planned to occur 48 hours after surgery (usually on the evening of 2nd postoperative day). Postoperative sagittal balance was 


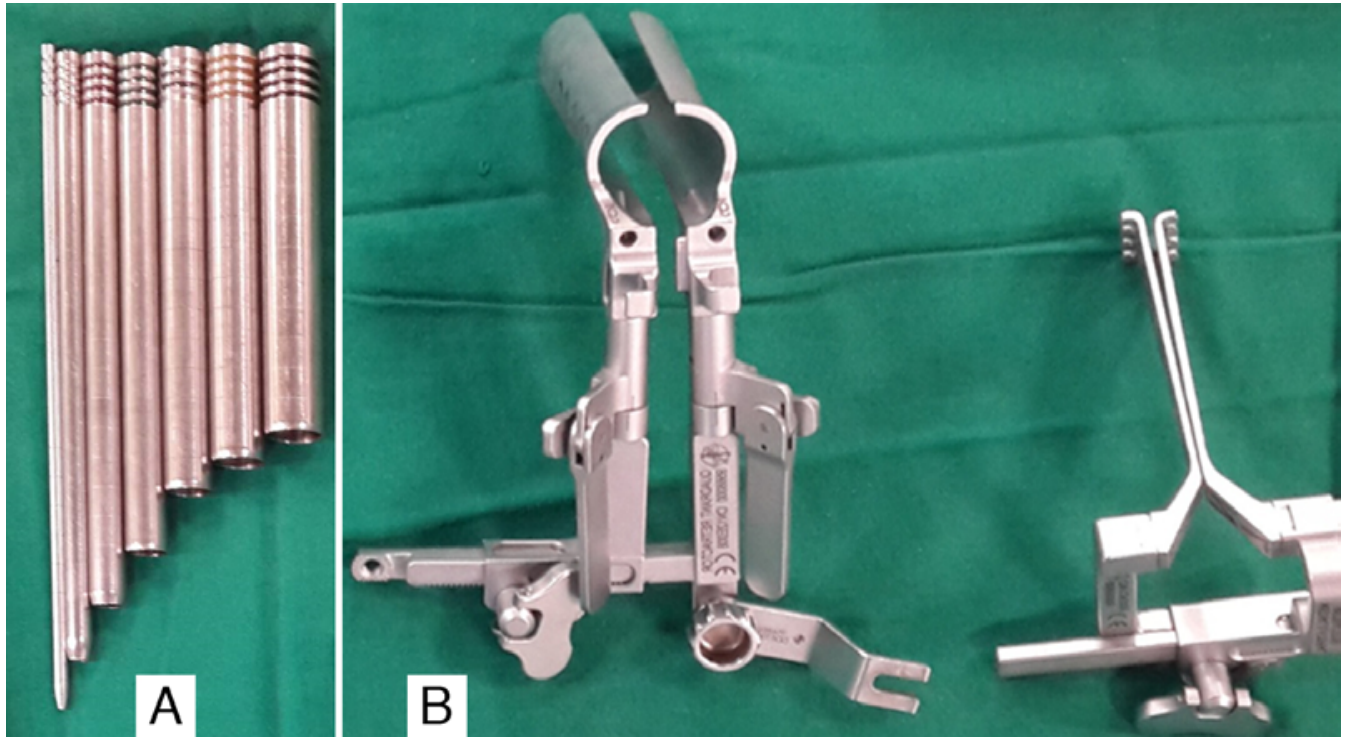

FIG. 2. Tubular dilators (A) and Quadrant retractor system with mediolateral retractor (B).

evaluated using radiography, and the degree of correction was noted with respect to the pelvic parameters LL, SS, PT, DSA, and PI.

Patients wore a lumbosacral belt for a minimum period of 2-3 weeks after surgery and were allowed to ambulate. At the end of that period, a graduated physiotherapy program was initiated for an average period of 3 months.

Data from subsequent follow-up visits after 1 month, 6 months, 1 year, and 2 years were recorded.

\section{Results}

A total of 36 consecutive patients with spondylolisthesis underwent minimally invasive TLIF. Information on age, sex, level of spondylolisthesis, and grade of slippage are summarized in Table 1. Symptoms included severe low-back pain, radicular pain in all patients with no bowel or bladder disturbance. Of the 36 levels of spondylolisthesis in 36 patients, 29 were Meyerding Grade II and 7 were Meyerding Grade III.

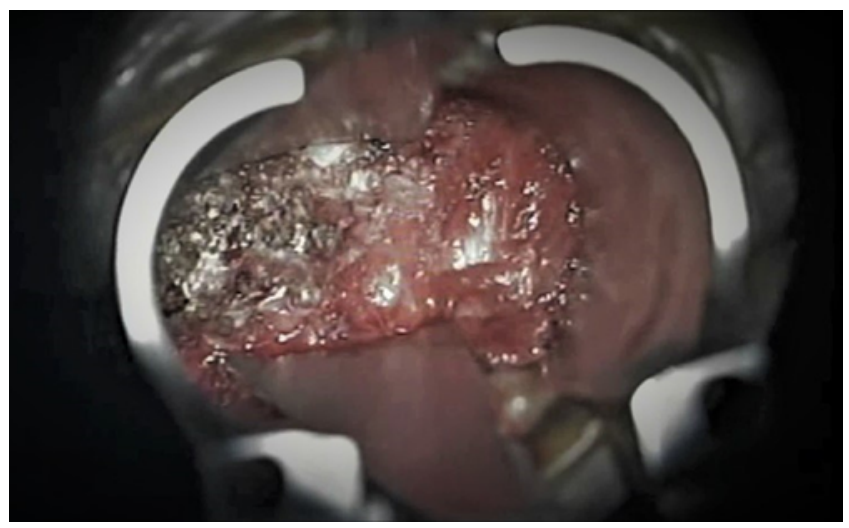

FIG. 3. Intraoperative view after retractor placement exposing field of interest-the facet joint and pars interarticularis.
No major perioperative complications occurred, and no conversion to an open procedure was required. The mean operative time (from skin incision to closure) was 146.7 minutes, and the mean estimated blood loss was approximately $90 \mathrm{ml}$. All patients were discharged on either the 2 nd or 3 rd postoperative day. The mean duration of follow-up was 30.4 months (range of 24-60 months).

\section{Clinical Outcomes}

At the most recent follow-up, 34 patients (94\%) were either pain free or had minimal pain (VAS score of $0-1$ ). There were 2 patients $(6 \%)$ who had moderate pain (VAS score of 2-5), which corresponded to a higher grade of spondylolisthesis. All patients had a significant improvement in pain scores $(\mathrm{p}<0.05)$. The mean preoperative VAS score for low-back pain was 6.5 (SD 1.5), whereas the mean 2-year follow-up postoperative score was 1.6 (SD 1.3). The mean preoperative ODI score was 53.7 (SD 13.1), whereas the mean 2-year follow-up postoperative ODI score was 22.5 (SD 15.5).

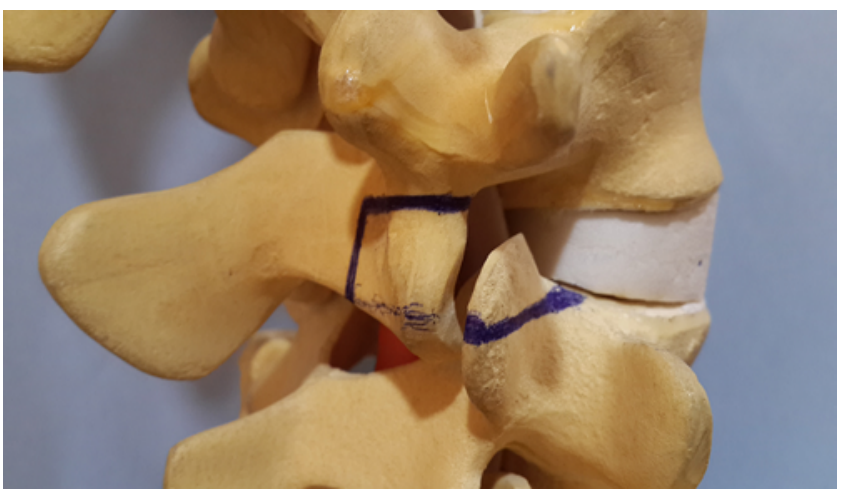

FIG. 4. Extent of facetectomy and bony chiseling to expose the disc space through Kambin's triangle. 


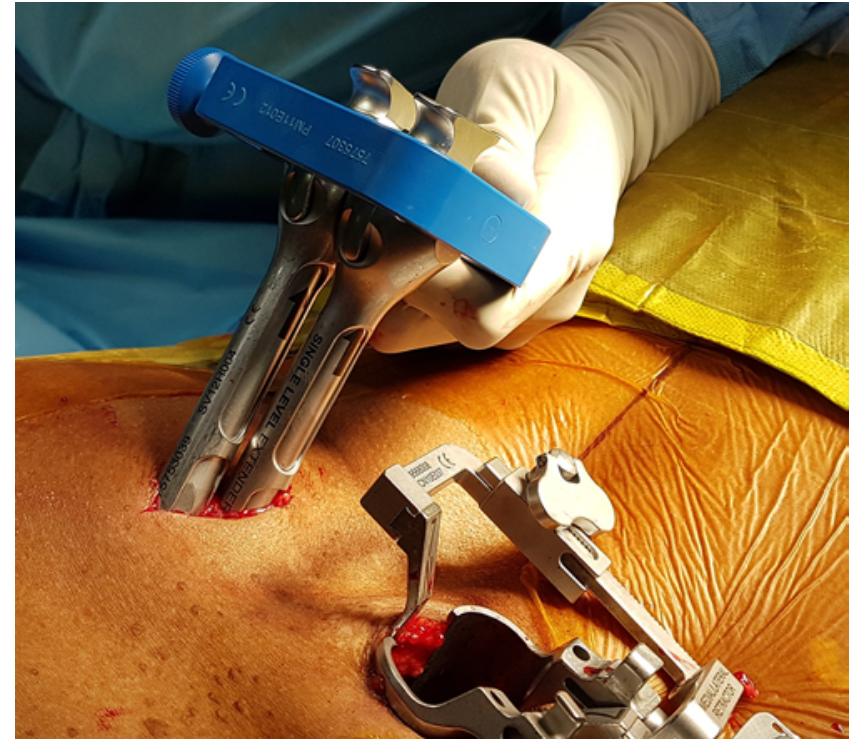

FIG. 5. After achieving adequate decompression, percutaneous screws are placed on the contralateral side and the Sextant towers are mated and held in place.

Table 2 summarizes the preoperative and 2-year postoperative clinical data.

\section{Radiological Outcomes}

Complete reduction of all grades of spondylolisthesis was achieved immediately after surgery in all 36 patients. The overall sagittal profile improved dramatically. Radiological parameters were found to improve with implants in situ at the 2-year follow-up examination. PI was the only parameter that remained relatively unchanged, whereas all the other parameters showed a significant change, especially DSA and LL.

In general, SS was found to be marginally decreased postoperatively ( $\mathrm{p}<0.001)$, while PT increased slightly but did not reach statistical significance. In 6 patients in whom the pelvis was "unbalanced," there was an increase in SS and a decrease in PT. Mean LL was significantly decreased, while mean DSA was significantly increased $(\mathrm{p}<0.001)$.

Examples of surgical cases are shown in Figs. 10-12.

Preoperative and 2-year follow-up radiological data are summarized in Table 3.

Anterior interbody fusion was graded according to Bridwell's classification, which assesses trabeculae formation, graft placement, and radiolucency (Table 4). Overall, fusion in $97 \%$ of the patients was "good" (Grades I and II) at the 2-year follow-up. In one patient with Grade III spondylolisthesis, fusion was delayed, with a mild loss of reduction at 6 months, but this was found to be stabilized at that position at subsequent follow-up examination. All other patients stabilized in the position that was fixed postoperatively.

There was a strong association between the improvement in the pelvic parameters and pain scores. When considering a clinical improvement in VAS scores in 34 of the
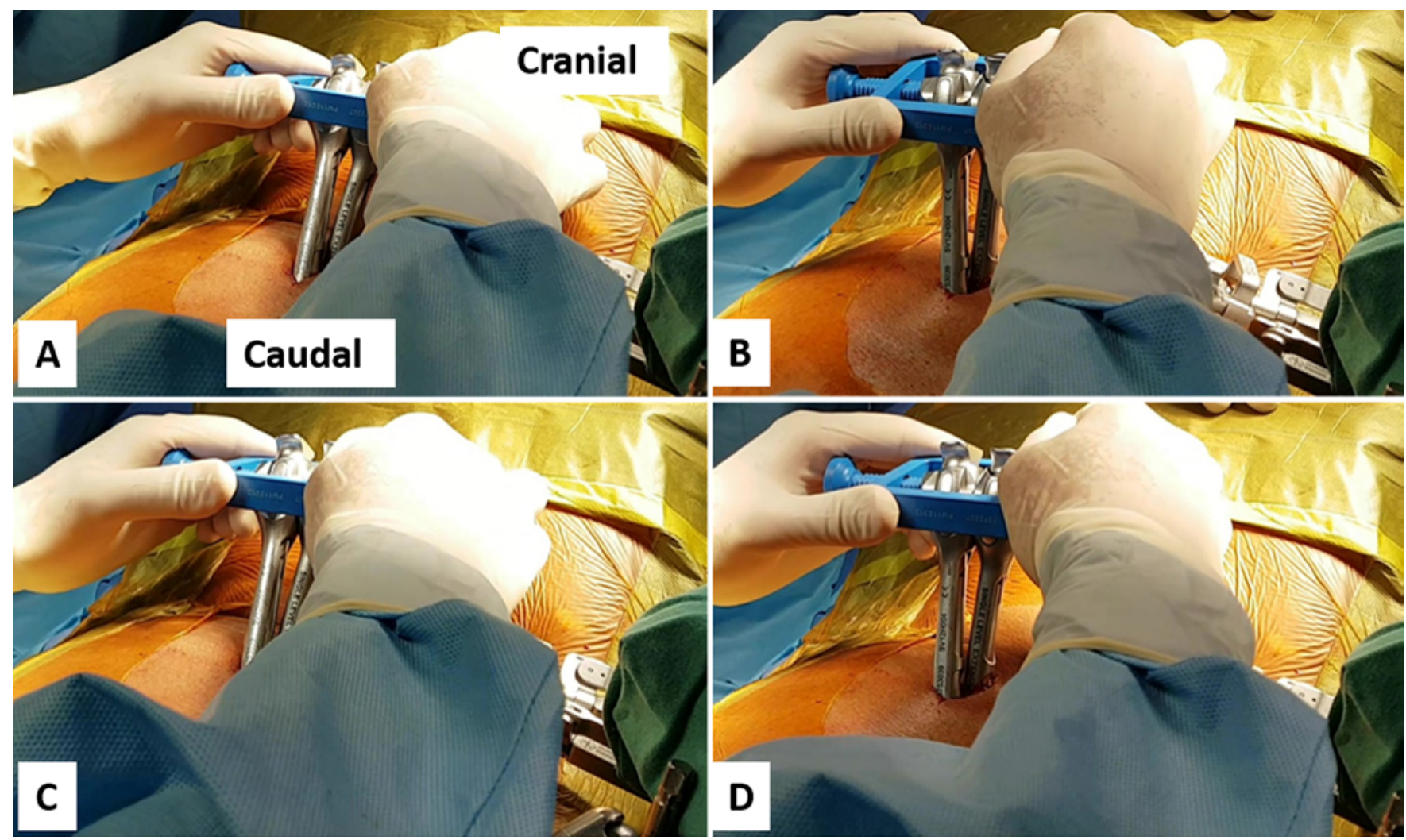

FIG. 6. A-D: Photographs in sequence showing the gentle back and forth "rocking" movement in the craniocaudal direction. 


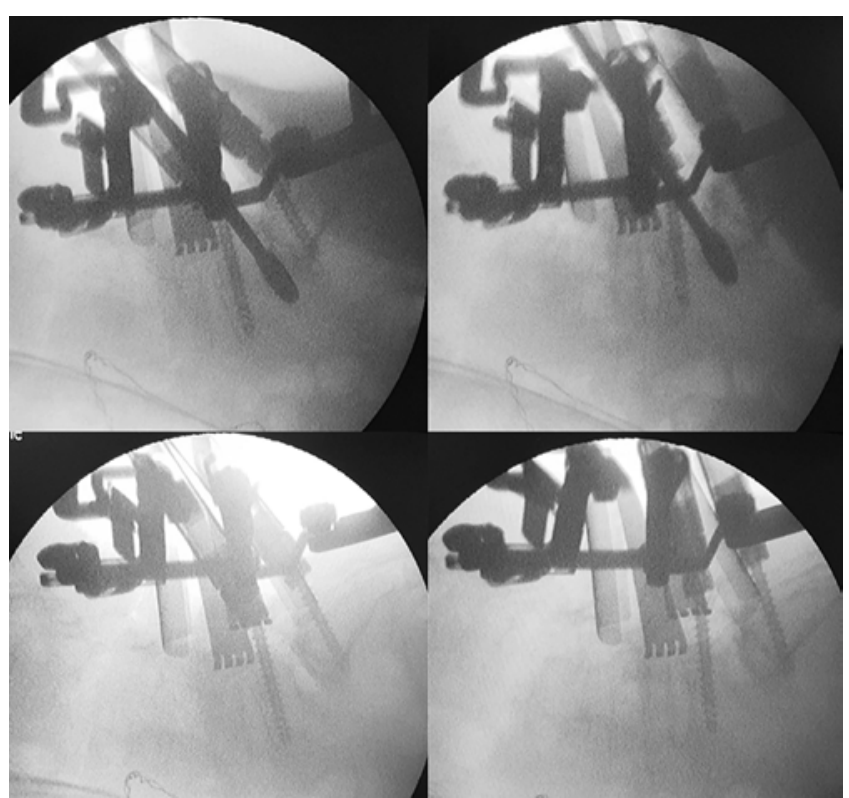

FIG. 7. Fluoroscopic images also demonstrating the "rocking" movement and gradual reduction of the spondylolisthesis.

36 patients, improvements in LL and DSA were noted in 30 patients. Using the Fisher exact test (0.02381 statistical value), we observed a very strong correlation $(\mathrm{p}<0.05)$ between radiological and clinical outcomes.

\section{Discussion}

\section{Sagittal Balance and Spondylolisthesis}

Using radiological imaging and patient-reported questionnaires, many studies have documented a correlation between sagittal imbalance and lumbosacral spine degeneration. In their 1998 article, Legaye et al. ${ }^{19}$ proposed and explained one anatomical parameter, the pelvic incidence, as the key factor for managing the spinal balance. A close relationship was observed between the PI and the SS, which strongly determines lumbar lordosis. A predictive equation was postulated, mathematically expressed as $\mathrm{PI}=\mathrm{SS}+\mathrm{PT}$.

In another major study, Labelle and colleagues ${ }^{17}$ analyzed sagittal alignment in developmental spondylolisthesis and found that PI, SS, PT, and LL were significantly greater in individuals with spondylolisthesis, with PI having a direct linear correlation with SS, PT, and LL. Increased PI appeared to be an important risk factor for development, as well as progression, of spondylolisthesis.

\section{Need for Surgical Reduction of Listhesis}

Much debate exists in the literature regarding the issue of reduction of high-grade spondylolisthesis. The question is whether partial or complete reduction should be performed in case of high-grade spondylolisthesis. A popular method of treatment has been in situ fusion, but studies have reported a high rate of pseudarthrosis, slip progression, and persistent cosmetic deformity. ${ }^{29}$ The advent of pedicle screws for fixation has brought about greater interest in reduction, but its effect on spinopelvic orientation remain poorly defined. Recent evidence indicates that re-

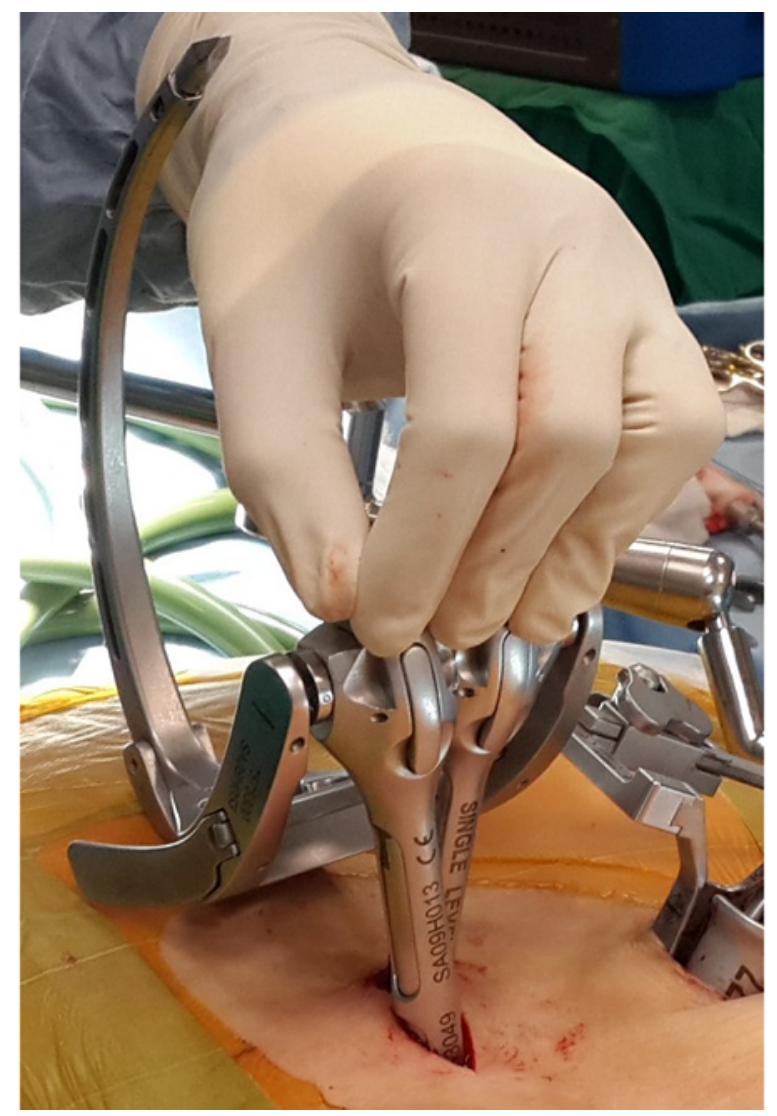

FIG. 8. Attachment of the Sextant arc for insertion of the pre-curved lordotic rod into the screw heads.

duction might be indicated for individuals with an unbalanced (retroverted or vertical) pelvis..$^{13}$

However, reduction of high-grade spondylolisthesis may be difficult, hazardous, and not always possible. In most cases, reduction is performed using open techniques as a general standard of care. In the past, various methods were used including, but not limited to, Harrington rod distraction, ${ }^{12}$ Bradford's cast reduction, ${ }^{4}$ and even recently using Magerl's external fixator with circumferential fusion. ${ }^{14}$ Also, an area of concern has been the need for extension of the fusion construct to involve adjacent healthy segments for improved stability. ${ }^{29}$

In a multicenter analysis, Labelle et al. ${ }^{18}$ studied changes in spinopelvic sagittal alignment after surgical correction of L5-S1 developmental spondylolisthesis, with the aim to determine the role of surgery in sagittal spinopelvic alignment. Their hypothesis was that surgical correction at the lumbosacral level was associated with an improvement in the overall shape of the spine and in the orientation of the pelvis. They found that pelvic shape was not affected by attempted surgical reduction. However, adequate repositioning of the slipped vertebrae significantly improved the overall pelvic balance and lumbar shape due to a reduction of the abnormally high lumbar lordosis and abnormal pelvic retroversion. The same was even more evident once the cases were divided into unbalanced and balanced pelvis groups. Thus, their results reiterated the need for reduction 

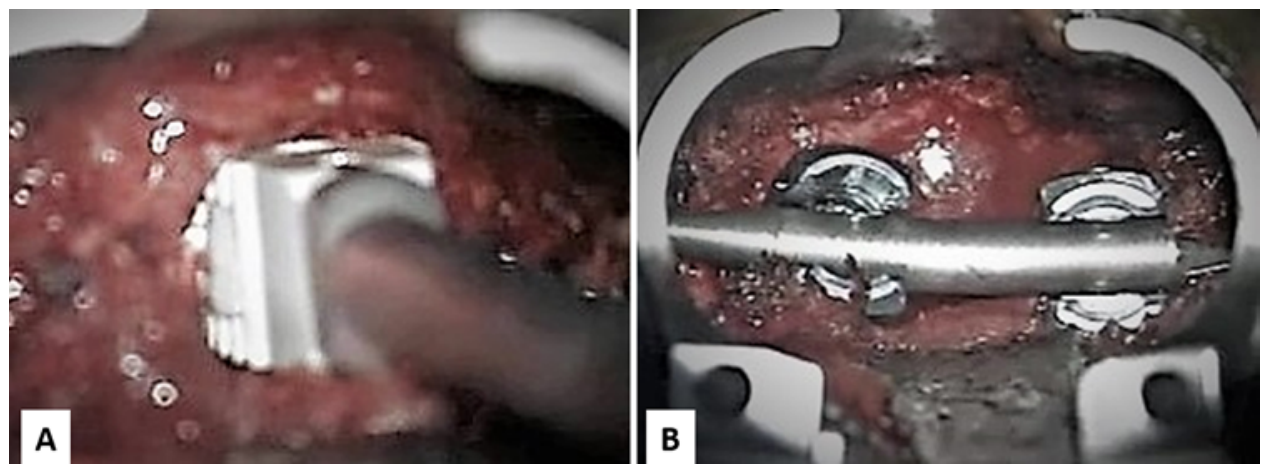

FIG. 9. Placement of the PEEK cage filled with autograft bone into the disc space (A) and the ipsilateral pedicle screws and rod under visualization (B).

techniques, especially in patients in whom the pelvis was unbalanced.

A more recent study by Bourghli et al. ${ }^{2}$ assessed the sagittal alignment of the spine and pelvis before and after surgical treatment of isthmic spondylolisthesis. They found significant improvement toward more normal values for PT and SS in relation to PI following surgery. Reduction combined with fusion of the slipped vertebral level was able to correct the anterior tilt with anterior sagittal imbalance due to spondylolisthesis, leading to restoration of the normal spinosacral angle. In those patients with insufficient restoration of sagittal balance parameters, outcomes were not as good as that of the others. It was thus concluded that surgical management of spondylolisthesis that restores correct values for the pelvic position-dependent parameters showed good clinical outcomes.

Schuller et al., ${ }^{26}$ analyzing sagittal spinopelvic alignment and facet joint degeneration in degenerative spondylolisthesis, found that the anatomical orientation of the

\section{TABLE 1. Patient demographic and spondylolisthesis data}

\begin{tabular}{cc}
\hline \multicolumn{1}{c}{ Factor } & No. of Cases $(\%)$ \\
\hline No. of patients & 36 \\
\hline Age in yrs & 51.7 \\
\hline Mean & $24-80$ \\
\hline Range & \\
\hline Sex & $14(39)$ \\
\hline M & $22(61)$ \\
\hline F & \\
\hline Meyerding grade & $29(80)$ \\
\hline II & $7(10)$ \\
\hline III & $4(11)$ \\
\hline Slippage level & $19(52)$ \\
\hline L3-4 & $13(36)$ \\
\hline L4-5 & \\
\hline L5-S1 & $15(42)$ \\
\hline Type of spondylolisthesis & $21(58)$ \\
\hline Isthmic &
\end{tabular}

pelvis with a high PI and SS was a predisposing factor for degenerative spondylolisthesis. Increasing PT suggested a posterior tilt of the pelvis as a compensation mechanism in patients with high PI. This would lead to an increase in the lordosis at the levels above the spondylolisthesis, which might subsequently increase posterior stress on facet joints.

Other studies suggest that the degree of the slip increases with the severity of disc degeneration; this in turn alters the DSA. As the vertebra progresses to slip or slide forward, the compensation mechanism adopted by the lumbar spine is to increase the lumbar lordosis, thereby increasing the wedging of the intervertebral disc. This morphological change leads to decreased DSA. ${ }^{23}$

Thus, there is a strong agreement that correction of the excess lumbar lordosis and restoration of a normal DSA by distraction would be an important surgical goal. Complete reduction and fixation with fusion halt the progression of spondylolisthesis in that particular level.

\section{Role of Minimally Invasive Surgery}

A search of the literature reveals that MIS-TLIF can be used in the treatment of low-grade spondylolisthesis with moderately good clinical results. ${ }^{15,22}$ Yet when it comes to higher grades of spondylolisthesis, there is a paucity of reports that address the use of MIS with reduction techniques.

It is already well known that MIS-TLIF can achieve similar or at times better clinical and radiological results than conventional open fusion in spondylolisthesis. ${ }^{7}$ Smaller skin incisions, reduced muscle disruption and denervation, very little neural handling, and decreased blood

TABLE 2. Summary of preoperative and 2-year follow-up pain scores

\begin{tabular}{|c|c|c|c|c|c|}
\hline \multirow[b]{3}{*}{ Measure } & \multicolumn{4}{|c|}{ Score } & \multirow{3}{*}{$\begin{array}{c}p \\
\text { Value* }^{*}\end{array}$} \\
\hline & \multicolumn{2}{|c|}{ Preop } & \multicolumn{2}{|c|}{ Postop } & \\
\hline & Mean & SD & Mean & SD & \\
\hline VAS & 6.5 & 1.5 & 1.6 & 1.3 & $<0.05$ \\
\hline ODI & 53.7 & 13.1 & 22.5 & 15.5 & $<0.05$ \\
\hline
\end{tabular}

* Statistical significance measured using the Wilcoxon test. 


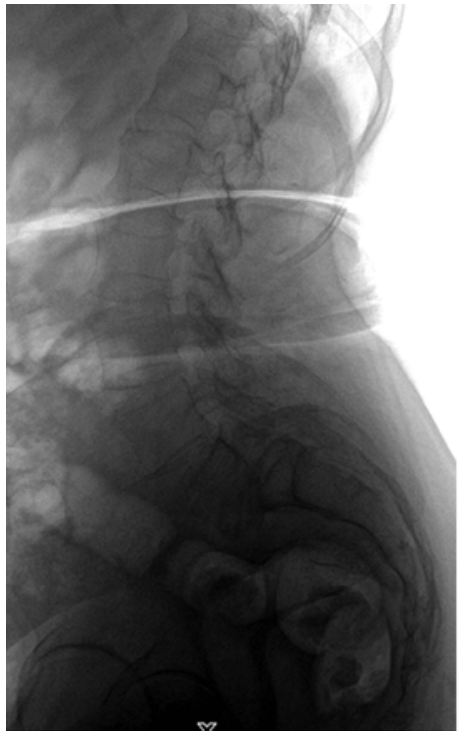

Pre-operative

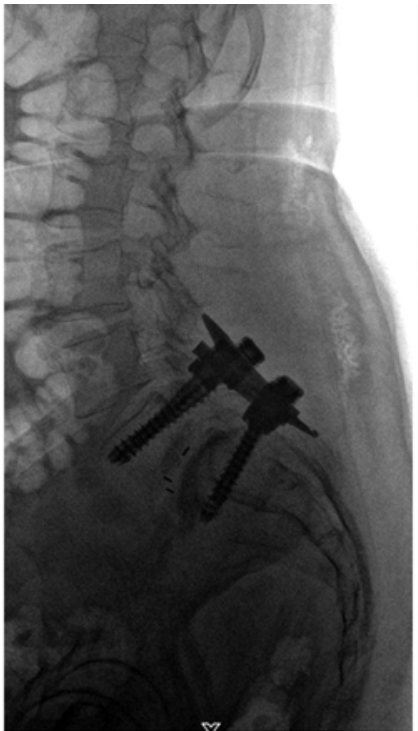

1 year

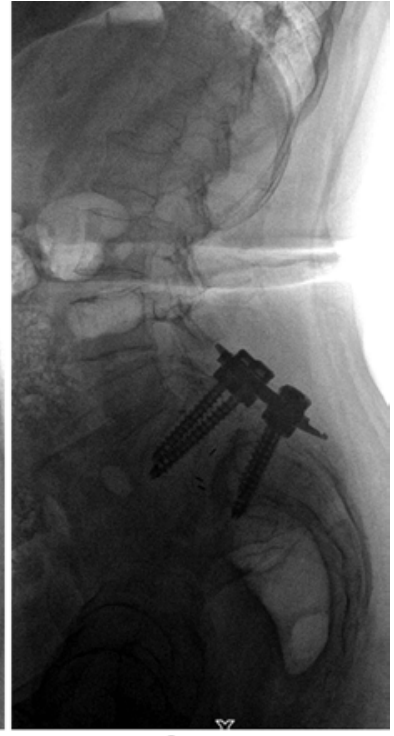

2 year

FIG. 10. Radiographs of a patient with Grade III spondylolisthesis. Good fusion is demonstrated.

loss, while at the same time providing good to excellent fusion results, are only some of the many proven advantages of a MIS-TLIF. ${ }^{11,24,33}$ Thus, MIS can bring about an overall reduction in the approach-related morbidity for the patient.

The goal of surgery in the present study was complete reduction of low- and high-grade spondylolisthesis by using MIS-TLIF. The use of specialized implants for the stabilization of spine and fixation of the reduction, along with a novel rocking technique, has helped to bring about successful correction of the segmental deformity that has led to an improvement of the overall sagittal profile. Our clinical and radiological outcomes further highlight the positive results by restoring a balanced posture and relieving radicular and lumbar pain.

This minimally invasive reduction technique relies on multiple steps: adequate facetectomy-even bilateral in

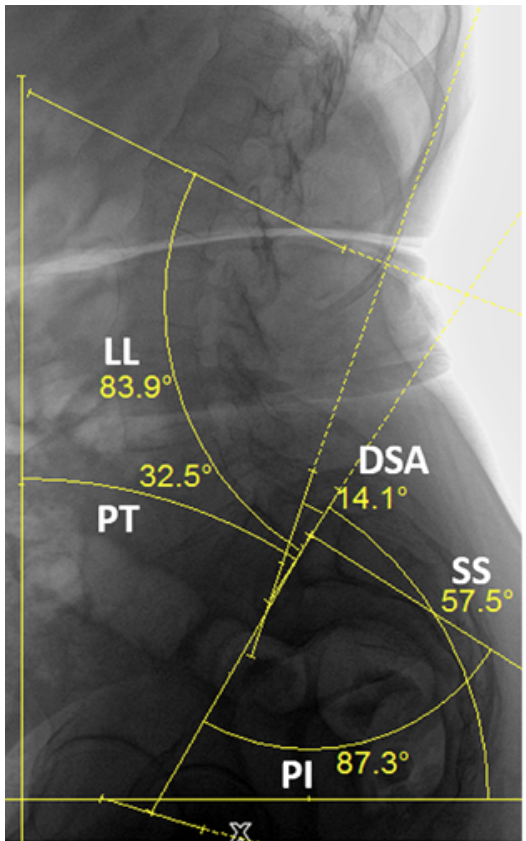

Pre-operative

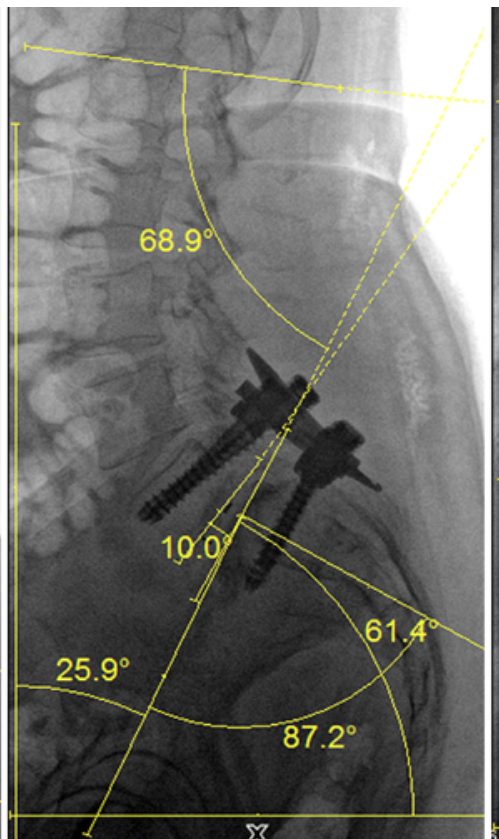

1 year

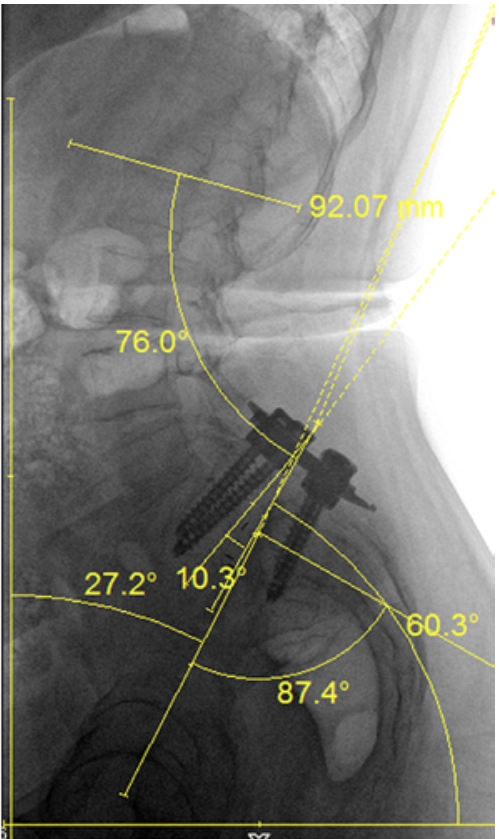

2 year

FIG. 11. Radiographs of the same patient showing measurement of radiological parameters. 


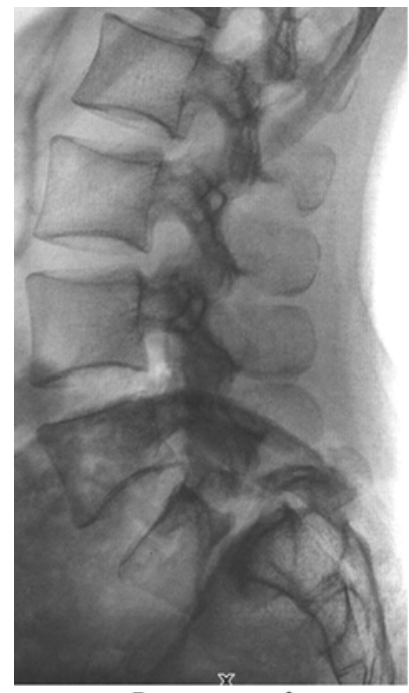

Pre-operative

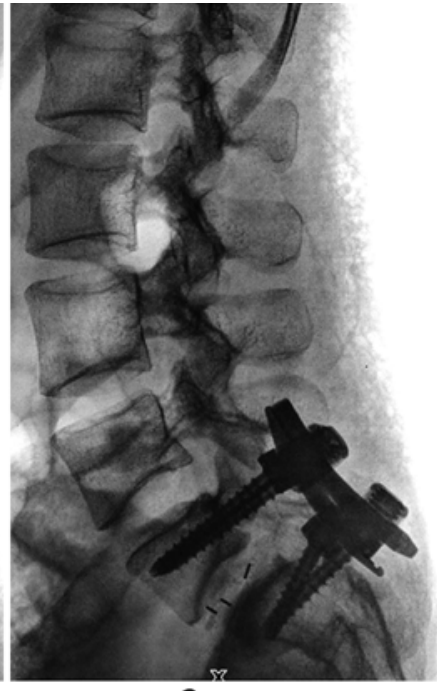

2 year
FIG. 12. Preoperative and postoperative radiographs of another patient with Grade III spondylolisthesis

occasional cases-good discectomy, and endplate preparation. These steps remain the main keystones for successful correction. The addition of the rocking technique is critical in reducing the spondylolisthesis such that complete anatomic reduction can be achieved on a consistent basis. The higher the grade of the spondylolisthesis, the greater is the degree of rocking that is required. Use of this "rocking" technique has surprisingly achieved reduction even when it appeared challenging in the higher grades of spondylolisthesis. The need for including adjacent-segmental support for reduction was not necessary in this series.

In one patient with Grade III spondylolisthesis, fusion occurred late, and in fact the patient was found to have a mild loss of reduction at follow-up. It is worth mentioning that no bone morphogenetic proteins were used in this study. It is believed that if bone morphogenetic proteins were to be used, especially in Grades 3 and higher spondylolisthesis, then fusion might occur faster and even the mild slip that occurred postoperatively in this patient might be avoided.

Furthermore, this study did not specifically assess patients with osteoporosis. None of the patients in the study

TABLE 3. Summary of preoperative and 2-year follow-up radiological data

\begin{tabular}{|c|c|c|c|c|c|}
\hline \multirow[b]{2}{*}{ Parameter } & \multicolumn{2}{|c|}{ Preop } & \multicolumn{2}{|c|}{ Postop } & \multirow{2}{*}{$\begin{array}{c}p \\
\text { Value }^{*}\end{array}$} \\
\hline & Mean & SD & Mean & SD & \\
\hline PT & 18 & 7.3 & 20.9 & 11.2 & 0.19 \\
\hline SS & 38.5 & 9.5 & 31.7 & 7.9 & $<0.001$ \\
\hline PI & 55 & 11.3 & 54.3 & 11.78 & 0.78 \\
\hline LL & 51.8 & 13.1 & 41.3 & 11.3 & $<0.001$ \\
\hline DSA & 6 & 5.2 & 9.9 & 3.9 & $<0.001$ \\
\hline
\end{tabular}

Mean and SD values are presented in degrees.

* Statistical significance measured using the paired t-test.
TABLE 4. Summary of anterior fusion grades according Bridwell's classification

\begin{tabular}{clc}
\hline Grade & \multicolumn{1}{c}{ Description } & $\begin{array}{c}\text { No. of } \\
\text { Patients }\end{array}$ \\
\hline I & Fusion w/ remodeling \& trabeculae & 23 \\
\hline II & Graft intact, not fully remodeled, no radiolucency & 12 \\
\hline III & Graft intact but a definite lucency & 1 \\
\hline IV & Definitely not fused, collapsed & 0 \\
\hline
\end{tabular}

group were found to have gross osteoporosis. However, the we reiterate that it would be very concerning to use this technique in patients with gross osteoporosis. Great caution is advocated in the use of this technique in the very elderly and osteoporotic patients because of the bone quality. In short, any technique of this sort, requiring rocking of the hardware and implants, would be preferable in the middle-aged or younger population rather than the elderly or in those with osteoporosis.

Nevertheless, this is a small cohort of patients with a single-center experience. The ability to use this technique in different centers should be explored. Greater numbers in higher degrees of spondylolisthesis will be needed to ensure the reliability of this technique. While our experience with minimally invasive surgery suggests promising results, open techniques must still be considered as the backbone of surgery for higher grades of spondylolisthesis and challenging situations.

\section{Conclusions}

Minimally invasive monosegmental TLIF for reduction using this rocking technique is effective in the treatment of various grades of spondylolisthesis. Complete reduction of the slippage and excellent correction of overall sagittal profile can be achieved, and the need to include any healthy adjacent segment in the fusion construct can be avoided.

\section{Acknowledgments}

We would like to acknowledge Clare Claiton, MSc, for her help in preparation of the manuscript.

\section{References}

1. Bernhardt M, Bridwell KH: Segmental analysis of the sagittal plane alignment of the normal thoracic and lumbar spines and thoracolumbar junction. Spine (Phila Pa 1976) 14:717721, 1989

2. Bourghli A, Aunoble S, Reebye O, Le Huec JC: Correlation of clinical outcome and spinopelvic sagittal alignment after surgical treatment of low-grade isthmic spondylolisthesis. Eur Spine J 20 (Suppl 5):663-668, 2011

3. Boxall D, Bradford DS, Winter RB, Moe JH: Management of severe spondylolisthesis in children and adolescents. J Bone Joint Surg Am 61:479-495, 1979

4. Bradford DS: Treatment of severe spondylolisthesis. A combined approach for reduction and stabilization. Spine (Phila Pa 1976) 4:423-429, 1979

5. Bridwell KH, Lenke LG, McEnery KW, Baldus C, Blanke $\mathrm{K}$ : Anterior fresh frozen structural allografts in the thoracic 
and lumbar spine. Do they work if combined with posterior fusion and instrumentation in adult patients with kyphosis or anterior column defects? Spine (Phila Pa 1976) 20:1410 1418,1995

6. Dai LY: Disc degeneration in patients with lumbar spondylolysis. J Spinal Disord 13:478-486, 2000

7. Dhall SS, Wang MY, Mummaneni PV: Clinical and radiographic comparison of mini-open transforaminal lumbar interbody fusion with open transforaminal lumbar interbody fusion in 42 patients with long-term follow-up. J Neurosurg Spine 9:560-565, 2008

8. Fairbank JC, Pynsent PB: The Oswestry Disability Index. Spine (Phila Pa 1976) 25:2940-2952, 2000

9. Farfan HF, Huberdeau RM, Dubow HI: Lumbar intervertebral disc degeneration: the influence of geometrical features on the pattern of disc degeneration--a post mortem study. J Bone Joint Surg Am 54:492-510, 1972

10. Hanson DS, Bridwell KH, Rhee JM, Lenke LG: Correlation of pelvic incidence with low- and high-grade isthmic spondylolisthesis. Spine (Phila Pa 1976) 27:2026-2029, 2002

11. Hari A, Krishna M, Rajagandhi S, Rajakumar DV: Minimally invasive transforaminal lumbar interbody fusion-indications and clinical experience. Neurol India 64:444-454, 2016

12. Harrington PR, Tullos HS: Spondylolisthesis in children. Observations and surgical treatment. Clin Orthop Relat Res 79:75-84, 1971

13. Hresko MT, Labelle H, Roussouly P, Berthonnaud E: Classification of high-grade spondylolistheses based on pelvic version and spine balance: possible rationale for reduction. Spine (Phila Pa 1976) 32:2208-2213, 2007

14. Karampalis C, Grevitt M, Shafafy M, Webb J: High-grade spondylolisthesis: gradual reduction using Magerl's external fixator followed by circumferential fusion technique and long-term results. Eur Spine J 21 (Suppl 2):S200-S206, 2012

15. Kim JS, Jung B, Lee SH: Instrumented minimally invasive spinal-transforaminal lumbar interbody fusion (MIS-TLIF); minimum 5-years follow-up with clinical and radiologic outcomes. J Spinal Disord Tech, 2012

16. Klineberg E, Schwab F, Smith JS, Gupta MC, Lafage V, Bess S: Sagittal spinal pelvic alignment. Neurosurg Clin N Am 24:157-162, 2013

17. Labelle H, Roussouly P, Berthonnaud E, Transfeldt E, O'Brien M, Chopin D, et al: Spondylolisthesis, pelvic incidence, and spinopelvic balance: a correlation study. Spine (Phila Pa 1976) 29:2049-2054, 2004

18. Labelle H, Roussouly P, Chopin D, Berthonnaud E, Hresko T, O’Brien M: Spino-pelvic alignment after surgical correction for developmental spondylolisthesis. Eur Spine J 17:11701176,2008

19. Legaye J, Duval-Beaupère G, Hecquet J, Marty C: Pelvic incidence: a fundamental pelvic parameter for three-dimensional regulation of spinal sagittal curves. Eur Spine J 7:99-103, 1998

20. Marty C, Boisaubert B, Descamps H, Montigny JP, Hecquet $\mathrm{J}$, Legaye J, et al: The sagittal anatomy of the sacrum among young adults, infants, and spondylolisthesis patients. Eur Spine J 11:119-125, 2002

21. Meyerding HW: Spondylolisthesis: surgical treatment and results. Surg Gynecol Obstet 54:371-377, 1932

22. Mummaneni PV: Percutaneous transforaminal lumbar interbody fusion for the treatment of degenerative lumbar instability. Neurosurgery 62:E1384, 2008

23. Oh YM, Eun JP: The relationship between sagittal spinopelvic parameters and the degree of lumbar intervertebral disc degeneration in young adult patients with low-grade spondylolytic spondylolisthesis. Bone Joint J 95-B:1239-1243, 2013
24. Rampersaud YR, Gray R, Lewis SJ, Massicotte EM, Fehlings MG: Cost-utility analysis of posterior minimally invasive fusion compared with conventional open fusion for lumbar spondylolisthesis. SAS J 5:29-35, 2011

25. Roussouly P, Gollogly S, Berthonnaud E, Labelle H, Weidenbaum M: Sagittal alignment of the spine and pelvis in the presence of L5-S1 isthmic lysis and low-grade spondylolisthesis. Spine (Phila Pa 1976) 31:2484-2490, 2006

26. Schuller S, Charles YP, Steib JP: Sagittal spinopelvic alignment and body mass index in patients with degenerative spondylolisthesis. Eur Spine J 20:713-719, 2011

27. Seitsalo S, Schlenzka D, Poussa M, Österman K: Disc degeneration in young patients with isthmic spondylolisthesis treated operatively or conservatively: a long-term follow-up. Eur Spine J 6:393-397, 1997

28. Toy JO, Tinley JC, Eubanks JD, Qureshi SA, Ahn NU: Correlation of sacropelvic geometry with disc degeneration in spondylolytic cadaver specimens. Spine (Phila Pa 1976) 37:E10-E15, 2012

29. Transfeldt EE, Mehbod AA: Evidence-based medicine analysis of isthmic spondylolisthesis treatment including reduction versus fusion in situ for high-grade slips. Spine (Phila Pa 1976) 32 (19 Suppl):S126-S129, 2007

30. Vedantam R, Lenke LG, Keeney JA, Bridwell KH: Comparison of standing sagittal spinal alignment in asymptomatic adolescents and adults. Spine (Phila Pa 1976) 23:211-215, 1998

31. Wang MY: Improvement of sagittal balance and lumbar lordosis following less invasive adult spinal deformity surgery with expandable cages and percutaneous instrumentation. J Neurosurg Spine 18:4-12, 2013

32. Wiltse LL, Widell EH Jr, Jackson DW: Fatigue fracture: the basic lesion is inthmic spondylolisthesis. J Bone Joint Surg Am 57:17-22, 1975

33. Wu JC, Mummaneni PV: Using lumbar interspinous anchor with transforaminal lumbar interbody fixation. World Neurosurg 73:471-472, 2010

\section{Disclosures}

The authors report no conflict of interest concerning the materials or methods used in this study or the findings specified in this paper.

\section{Author Contributions}

Conception and design: Rajakumar, Krishna. Acquisition of data: Rajakumar, Hari, Sharma, Reddy. Analysis and interpretation of data: Rajakumar, Hari. Drafting the article: Rajakumar, Hari. Critically revising the article: Rajakumar, Hari, Krishna. Reviewed submitted version of manuscript: Rajakumar, Hari, Krishna. Approved the final version of the manuscript on behalf of all authors: Rajakumar. Statistical analysis: Rajakumar, Hari. Administrative/technical/material support: Rajakumar, Hari, Sharma, Reddy. Study supervision: Rajakumar, Hari, Krishna.

\section{Supplemental Information Videos \\ Video 1. https://vimeo.com/221725711. \\ Video 2. https://vimeo.com/221725975.}

\section{Correspondence}

Deshpande V. Rajakumar, Department of Neurosurgery, Fortis Hospitals, \#154/9, Opp. IIM-B, Bannerghatta Rd., Bangalore 560076, India.email: drajakumar9@gmail.com. 